\title{
Changes in nocturnal oximetry after treatment of exacerbations in cystic fibrosis
}

\author{
M B Allen, A F Mellon, E J Simmonds, R L Page, J M Littlewood
}

\begin{abstract}
Sleep related arterial oxygen desaturation has been described in clinically stable young adults with cystic fibrosis. The incidence and severity of nocturnal oxygen desaturation in children during infective exacerbations and the changes that occur with treatment were examined. Forty five children with proved cystic fibrosis, median age 8.9 years, admitted to the Regional Cystic Fibrosis Unit underwent clinical evaluation, spirometry, and measurement of peak flow and nocturnal oxygen saturation on admission and after 10 days' treatment.

There was a significant improvement in all the above measurements, with the averaged overnight saturation changing from a mean (SD) $92.7(2.7) \%$ to 94.3 $(2 \cdot 0) \%$, mean (SE) difference $1.58(0 \cdot 37)$. The time spent with a saturation $4 \%$ or more below their clinic value showed a marked improvement from 122 (152) minutes on the first night to $21(30.7)$ on the second, mean (SE) difference 101 (22.4). Eight young children could not perform pulmonary function tests, all desaturated on the admission night.

Nocturnal hypoxaemia is a common finding in young cystic fibrosis patients during infective exacerbations but improves with treatment. Overnight oximetry is simple to perform, well tolerated, and identifies patients with marked nocturnal desaturation.

(Arch Dis Child 1993; 69: 197-201)
\end{abstract}

Pulmonary hypertension and cor pulmonale are common sequelae of the pulmonary disease found in cystic fibrosis. ${ }^{2}$ Those patients with advanced disease may show daytime hypoxaemia but some with normal awake arterial oxygen saturations develop hypoxaemia during sleep. ${ }^{3}$ The largest falls in saturation occur during rapid eye movement (REM) sleep and relate to reductions in functional residual capacity and episodic hypoventilation. ${ }^{4} 5$ Those with the greatest falls in nocturnal arterial oxygen saturation are the most likely to have pulmonary hypertension. 235

Several studies examining the relationship between sleep and nocturnal hypoxaemia in cystic fibrosis have been published. However, these patients have been clinically stable when studied and relatively old, with a mean age ranging between 15 and 20 years. ${ }^{4-12}$ There have been no studies investigating the extent of nocturnal desaturation in young children, the effect of infective exacerbations of their pulmonary disease on time spent desaturated during sleep, and the changes in saturation which occur with treatment.

\section{Aims and methods}

In an attempt to document the extent of sleep related hypoxaemia during infective exacerbations and changes with treatment we performed nocturnal oximetry on admission and after 10 days. In addition the relationship between nocturnal desaturation on admission and other clinical measurements were examined.

Children with confirmed cystic fibrosis admitted to the Regional Cystic Fibrosis Unit with infective exacerbations of their pulmonary disease requiring parenteral antibiotics were studied. Patients were enrolled over a 14 month period; those admitted in cor pulmonale or previously studied were excluded. The Shwachman ${ }^{13}$ and ChrispinNorman $^{14}$ scores and the resting oxygen saturations when well were obtained from recent clinic attendances.

On admission and at day 10 the forced expired volume in one second $\left(\mathrm{FEV}_{1}\right)$ and forced vital capacity (FVC) were obtained before bronchodilator treatment from the best of three forced expiratory manoeuvres (Vitalograph Compact) along with the peak expiratory flow rate (Wright's peak flow meter). All patients had their height measured to allow the pulmonary function results to be expressed as a percentage of predicted value. A clinical score based on the patients' symptoms and signs that we use routinely was also noted at these time periods. ${ }^{1516}$

Overnight oximetry using the Biox 3740 pulse oximeter (Ohmeda) was obtained on admission and after 10 days' treatment. A finger probe was attached between 2200-2300 hours and removed eight hours later. The children were observed by trained nursing staff throughout the study period. The Biox 3740 oximeter has the facility to store eight hours of data and its use in recording overnight saturation has been verified. ${ }^{17}$ Finger clubbing, present in a few older children, does not affect oximetry results. ${ }^{18}$

Oxygen saturation and heart rate were displayed on a compressed time base and one investigator (MBA) inspected the traces for errors and evidence of upper airway obstruction. ${ }^{19}$ The stored oximeter signal was also analysed by a purpose written 'in house' computer program, which rejected spurious desaturation due to movement artefact and 
calculated the average saturation and the lowest value; the awake supine saturation was recorded by nursing staff. The patient's daytime saturation when well, obtained from clinic, was entered into the computer to calculate the time spent desaturated, defined as the time spent at a saturation of $4 \%$ or more below their normal clinic value. ${ }^{19-22}$ Clinically significant desaturation was said to have occurred if time spent desaturated was greater than five minutes.

The children were all treated with intravenous antibiotics, bronchodilators, and physiotherapy. None received supplemental oxygen treatment. Eight were given reducing doses of prednisolone while an additional three patients continued with their inhaled steroids.

Depending on the normality of distribution, results are expressed as median (interquartile range, IQR) and mean (SD) to show the spread. Changes between results over the two study periods are shown as the mean differences and SE of this mean. The significance of any changes were calculated using parametric and non-parametric tests, with $\mathrm{p}=0.05$ taken as significant. Correlations between data were examined using Spearman's rank correlation test. The study was approved by the Leeds Eastern Health Authority ethical committee.

\section{Results}

(1) PATIENTS AND CLINICAL CONDITION Forty five children, 27 girls and 18 boys, with a median (IQR) age of $8.9(4.4-11 \cdot 2)$ years were enrolled. There was a wide range of disease severity with Shwachman scores ranging between 40 and 100, median 80 . The duration of hospital stay varied from 11 to 21 days, with a median of 14 days.

Ten children were unable to perform tests of pulmonary function reliably on both occasions: two because they were so unwell initially and in eight because of age; therefore paired data are available for 35 patients. Results are expressed as percentages of the predicted values, a valid method of assessment given that the height to age ratio for the patients had a mean value of $96 \%$.

There were improvements in pulmonary function after treatment, as shown by the significant changes in peak flow, $\mathrm{FEV}_{1}$, and FVC displayed in table 1 . The median (IQR) clinical score on admission fell significantly $(\mathrm{p}<0.001)$ after treatment from $7(2-15)$ to 3 $(0-11)$.

Table 1 Changes in percentage predicted (\% pred) spirometry, peak flow, and averaged nocturnal saturation ( $\mathrm{SaO}_{2}$ ) for all patients on both study periods

\begin{tabular}{|c|c|c|c|c|c|}
\hline & $\begin{array}{l}\text { No of } \\
\text { patients }\end{array}$ & $\begin{array}{l}\text { First study } \\
\text { mean }(S D)\end{array}$ & $\begin{array}{l}\text { Second study } \\
\text { mean }(S D)\end{array}$ & $\begin{array}{l}\text { Mean (SE) } \\
\text { difference }\end{array}$ & p Value ${ }^{\star}$ \\
\hline $\mathrm{FEV}_{1}(\%$ pred $)$ & 35 & $65 \cdot 0(20 \cdot 5)$ & $74.0(18.4)$ & $8 \cdot 6(2 \cdot 2)$ & $<0.001$ \\
\hline FVC ( $\%$ pred) & 35 & $84 \cdot 7(23 \cdot 6)$ & $97.6(18.5)$ & $13 \cdot 1(3 \cdot 0)$ & $<0.001$ \\
\hline Peak flow (\% pred) & 35 & $85.9(19 \cdot 1)$ & $101 \cdot 8(21 \cdot 1)$ & $15 \cdot 3(2 \cdot 6)$ & $<0.001$ \\
\hline Awake supine $\mathrm{SaO}_{2}(\%)$ & 45 & $91 \cdot 4(3 \cdot 0)$ & $93 \cdot 0(3 \cdot 1)$ & $1.6(0.5)$ & $<0.001$ \\
\hline Averaged nocturnal & & & & & \\
\hline $\mathrm{SaO}_{2}(\%)$ & 45 & $92 \cdot 7(2 \cdot 7)$ & $94 \cdot 3(2 \cdot 0)$ & $1 \cdot 6(0 \cdot 4)$ & $<0.001$ \\
\hline $\mathrm{Minimum} \mathrm{SaO}_{2}(\%)$ & 45 & $84 \cdot 5(5 \cdot 4)$ & $87 \cdot 0(4 \cdot 2)$ & $1.5(0.9)$ & NS \\
\hline $\begin{array}{l}\text { Time spent } \\
\text { desaturated (min) }\end{array}$ & 45 & $122 \cdot 0(152 \cdot 0)$ & $21 \cdot 0(30 \cdot 7)$ & $101 \cdot 0(22 \cdot 4)$ & $<0.001$ \\
\hline
\end{tabular}

\section{(2) OXYGEN SATURATION: CHANGES WITH} TREATMENT

Satisfactory results were obtained from all 45 patients, regardless of age; none had sleep apnoea. The mean clinic saturation of the 45 patients was $95 \cdot 2 \%(1 \cdot 6)$. After treatment the supine awake saturation and the averaged nocturnal saturation showed significant improvements, although the minimum saturation recorded was not different, as shown in table 1 .

Thirty eight patients showed evidence of clinically significant sleep related desaturation (five minutes or longer spent with a saturation $4 \%$ or more below their normal clinic value) on the first study, decreasing to 34 on the second night. Seven children showed greater desaturation on day 10 of the study, although in two this was trivial. Of the remaining five patients the changes could be partially explained by the development of resistant organisms, a viral infection, and/or bronchospasm on the second study night. The changes in time spent desaturated between the two study nights for all 45 children are shown graphically in the figure.

There was a marked change in the time spent desaturated for the whole group after treatment, falling from 122 (152) minutes initially to $21(30.7)$ minutes after treatment; mean (SE) difference $101(22 \cdot 4) ; \mathrm{p}<0.001$. The changes in magnitude of nocturnal desaturation can be further examined by arbitrarily dividing the patients into two groups using a cut off of more or less than 100 minutes clinically significantly desaturated. During the first study 17 patients were desaturated for over 100 minutes falling to two during the second study $\left(\chi^{2}=15, \mathrm{p}<0.001\right)$.

\section{(3) OXYGEN SATURATION: IDENTIFICATION OF} CHILDREN WHO DESATURATE

To examine the relationship between awake supine saturation and time spent desaturated the children were divided into two groups on the basis of their admission awake saturation. Two 'cut off' values were used: (a) less than $93 \%$ or $93 \%$ and above and (b) less than $92 \%$ or $92 \%$ and above. The mean (SD) results for awake saturation, time spent significantly

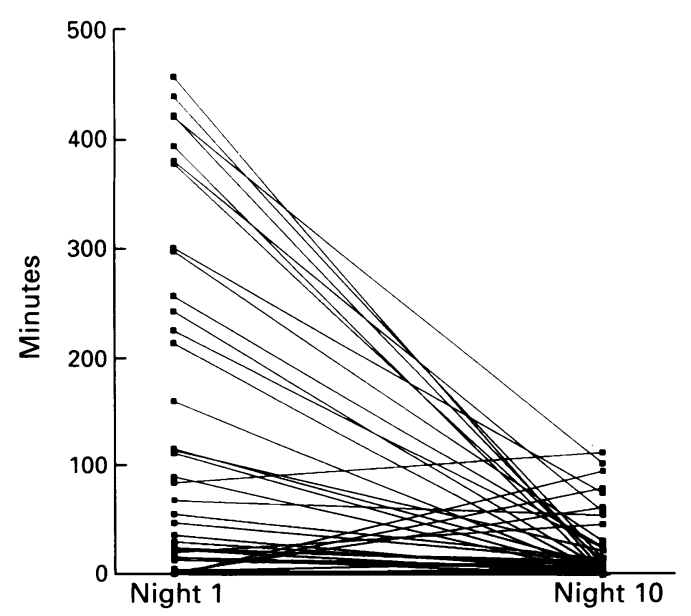

Changes in time spent desaturated for all patients between the admission night and after 10 days' standard treatment. 
Table 2 Mean (SD) for the initial supine awake saturation, time spent significantly desaturated, averaged nocturnal saturation $\left(\mathrm{SaO}_{2}\right)$ and percentage predicted (\% pred) spirometry for all children divided into two groups on the basis of their awake supine oximetry

\begin{tabular}{lllll}
\hline & $\mathrm{SaO}_{2} \geqslant 93 \%$ & $\mathrm{SaO}_{2}<93 \%$ & $\mathrm{SaO}_{2} \geqslant 92 \%$ & $\mathrm{SaO}_{2}<92 \%$ \\
\hline No of children & 16 & 29 & 22 & 23 \\
Supine awake $\mathrm{SaO}_{2}(\%)$ & $94 \cdot 7(1 \cdot 2)$ & $89 \cdot 5(2 \cdot 0)$ & $93 \cdot 9(1 \cdot 6)$ & $88 \cdot 9(1 \cdot 7)$ \\
Initial time desaturated (min) & $14 \cdot 6(21 \cdot 2)$ & $181 \cdot 2(160 \cdot 5)$ & $38 \cdot 5(70 \cdot 3)$ & $201 \cdot 8(166 \cdot 6)$ \\
Averaged nocturnal $\mathrm{SaO}_{2}(\%)$ & $95 \cdot 1(0 \cdot 9)$ & $91 \cdot 4(2 \cdot 5)$ & $94 \cdot 4(2 \cdot 1)$ & $91 \cdot 1(2 \cdot 3)$ \\
No of children & 13 & 22 & 17 & 18 \\
$\mathrm{FEV}_{1}(\%$ pred) & $70 \cdot 3(16 \cdot 5)$ & $61 \cdot 9(22 \cdot 3)$ & $70 \cdot 3(20 \cdot 1)$ & $60 \cdot 6(20 \cdot 2)$ \\
\hline
\end{tabular}

desaturated, averaged saturation and the initial spirometry are shown in table 2. Age, initial clinical score, Shwachman and ChrispinNorman scores, peak flow, clinic saturation, and minimum saturation recorded were not significantly different and are not included.

An awake saturation of $92 \%$ was a reasonable cut off with 22 patients whose arterial oxygen saturation was above this value spending 38.5 minutes desaturated. The figure of $93 \%$ is a better cut off with 16 children in this group spending only 14.6 minutes desaturated. In contrast the 29 children whose awake saturation was below $93 \%$ spent $181 \cdot 2$ minutes significantly desaturated. If the results of the 16 children are examined more closely, 10 showed no significant desaturation while the remaining six spent between 15 and 68 minutes desaturated. These six patients could not be identified by any other measurements.

\section{(4) RELATIONSHIP BETWEEN DESATURATION AND DISEASE SEVERTYY}

There was no significant correlation (Spearman's) between the time spent desaturated on the first night and age, peak flow, spirometry, Shwachman, Chrispin-Norman, or initial clinical scores. Furthermore, there was no correlation between the change in spirometry and improvement in time spent desaturated.

If the group of 17 children who spent over 100 minutes desaturated are compared with the remaining 28 patients several significant differences emerge. The median (IQR) clinical score was worse in the hypoxaemic group at 8 $(6-10)$ against $6(4-8),(p=0 \cdot 025)$. The initial supine awake and initial minimum saturations were $88.8(2 \cdot 1) \%$ and $81.4(6 \cdot 1) \%$, both significantly lower in the hypoxaemic children than in the other children at $92.9(2.4) \%$ and $86.4(3.8) \% ;(p<0.001$ and $p=0.001)$ respectively. Spirometry was obtained from 15 of the hypoxaemic children and 20 of the less hypoxaemic group. Although no differences were found in the admission spirometry between the two groups the post treatment values were significantly worse in the more hypoxaemic group. The $\mathrm{FEV}_{1}$, as percentage predicted, was $67(16.4) \%$ in the hypoxaemic group and $78.8(18.4) \%$ in the others $(p=0.048)$, with figures of $90.7(18.1)$ and $102.3(17 \cdot 7)$ being found for the FVC $(p=0.035)$. Age, Shwachman and ChrispinNorman scores were similar for the two groups.

Previous work in adolescents with clinically stable cystic fibrosis suggested that an $\mathrm{FEV}_{1}$ of below $65 \%$ predicted may identify those patients who develop sleep related desaturation. ${ }^{10}$ If this figure of $65 \%$ predicted is applied to the admission $\mathrm{FEV}_{1}$ in this study 15 children whose $\mathrm{FEV}_{1}$ was $82.5 \%(14.0)$ spent an average of 55.2 (110) minutes desaturated. In contrast the 19 patients whose first $\mathrm{FEV}_{1}$ was $51.4(13.8) \%$ spent 162.9 $(150 \cdot 4)$ minutes desaturated $(p=0 \cdot 05)$.

\section{(5) YOUNG CHILDREN}

Eight children whose median (IQR) age was $2 \cdot 7(2-4 \cdot 1)$ years were unable to perform spirometry because of their age and poor technique. All showed nocturnal hypoxaemia on the first study, with a mean (SD) time spent desaturated of 139.0 (168.0) minutes, improving to $28(38.3)$ minutes after treatment; mean (SE) difference 111.9 (62.4); $\mathrm{p}=0 \cdot 1$. The median (IQR) age of the remaining 37 children was $10.4(6 \cdot 7-11 \cdot 8)$ years and they spent similar periods significantly desaturated on the two study nights at $118.4(150 \cdot 4)$ and $19.5(29.2)$ minutes respectively. The averaged saturation for the eight children was $93(2 \cdot 3) \%$ on the first night and $95 \cdot 7(1 \cdot 7) \%$ on the second, while for the remaining 37 children the values were $92.6(2 \cdot 8) \%$ and 94 $(1.9) \%$ respectively. Only the results between the second nights were significantly different $(p=0.02)$ between the two groups.

The median (IQR) for the ChrispinNorman score in the young children was 3 $(2-5 \cdot 3)$, while a value of $7(4 \cdot 3-11 \cdot 8)$ was found in the older group $(p=0.006)$. The median Shwachman score in the eight young children was 85 (85-95) and 75 (65-85) in the 37 older children $(p=0 \cdot 013)$.

\section{Discussion}

Sleep related hypoxaemia has been found on full polysomnography in some adoloscents and young adults (age ranges 15-20 years) with cystic fibrosis when clinically stable. ${ }^{4-12}$ There have been no studies examining the extent of sleep related desaturation in younger patients and the effect of an infective exacerbation on any nocturnal desaturation that occurs. We have attempted to address these issues by documenting the desaturation found in 45 children (median age 8.9 years) during an exacerbation, the changes that occur with treatment, and the association between desaturation and other clinical measurements.

The study was ward based on sick children and therefore full polysomnography was not performed. An acclimatisation night, normally used in sleep studies, ${ }^{23}$ was not done as we wished to document the extent of any desaturation on the day of admission. REM sleep is the stage when the most profound desaturation occurs $^{45}$ and this is reduced on the first night of any sleep study. ${ }^{23}$ Therefore by not allowing patients a night to acclimatise we will have possibly underestimated the severity of any sleep related desaturation.

In previous studies the time spent desaturated in minutes has not been fully examined. Calculation of this from the 
subject's admission awake saturation would be inappropriate during infective exacerbations of pulmonary disease as one would anticipate the resting saturation to be lower than normal. On the study after treatment the awake saturation will probably have improved but may still not be back to normal for that child.

These assumptions are borne out by the normal clinic saturation of $95.2(1.6)$ being significantly greater than the supine awake saturation on admission 91.4 (3.0) and after treatment $93.0(3.1) ; \mathrm{p}<0.001$ and $\mathrm{p}<0.001$ respectively. The best method of determining the level from which desaturation occurs is to use a value when the child is well - the clinic saturation. Using this, the time spent desaturated on both study nights can be calculated and compared.

Clinically significant desaturation (defined as a saturation of $4 \%$ or below the normal (clinic) value for more than five minutes) was found in 38 children on admission and 34 after treatment. The large numbers who continue to desaturate after treatment can probably be explained by the delay in recovery of gas exchange, similar results occurring after infective exacerbations of pulmonary disease in patients with chronic bronchitis and emphysema. ${ }^{24}$ These results would be disappointing if the time spent desaturated was not examined as this showed a marked reduction, falling from 122 initially to $11 \cdot 7$ minutes after treatment (table 1 , figure).

Identification of individuals who have sleep related hypoxaemia during infective exacerbations without recourse to oximetry would be helpful. In clinically stable young adults the awake oxygen saturation and the lowest saturation on exercise failed to identify all subjects who desaturate during sleep, ${ }^{10} 12$ while regression analysis showed an $\mathrm{FEV}_{1}$ below $65 \%$ predicted to be useful. ${ }^{10}$ In the 45 children we studied age, peak flow, spirometry, Shwachman, Chrispin-Norman, and clinical scores on admission failed to identify children who desaturated. By subdividing the children into two groups on the basis of their admission $\mathrm{FEV}_{1}$ being above or below $65 \%$ predicted a barely significant difference in time spent desaturated was found.

To examine if the initial awake supine saturation could be used to predict nocturnal hypoxaemia the children were divided into two groups on the basis of their admission supine saturations. A saturation of $93 \%$ appears to be a good cut off with the 16 children whose awake saturation was $93 \%$ or greater spending only 14.6 minutes significantly desaturated. However, this result is misleading as 10 of the 16 children showed no desaturation on the first study and the remaining six spent an average of 38.9 minutes significantly desaturated. Furthermore, these children could not be identified by any other means.

An unexpected finding was the marked desaturation found in eight children, median age $2 \cdot 7$ years, who were too young to perform spirometry. Such severe hypoxaemia in young children during exacerbations is not widely recognised. Despite significantly better Shwachman and Chrispin-Norman scores, these eight children showed a similar magnitude or hypoxaemia as the other children on the first night, but a significantly better averaged saturation after treatment, possibly reflecting more rapid recovery.

We have shown for the first time in a large number of children with cystic fibrosis that sleep related oxygen desaturation is common during infective exacerbations, especially in young children with good Shwachman and Chrispin-Norman scores. When compared with the remaining children, those with prolonged desaturation appear to have lower spirometry results after treatment, a clinical score on admission that is worse, and lower initial awake saturations.

Unfortunately when examining the group as a whole, routine clinical measurements failed to detect children who develop nocturnal desaturation. It would have been expected that the awake supine saturation would be a useful predictor; however, six of the children whose saturation was $93 \%$ or above continued to desaturate during sleep.

This study highlights the usefulness of nocturnal oximetry in identifying sleep related desaturation. It was well tolerated, simple to perform, and provided information that could not be obtained by other measurements, especially in those children too young to perform reliably spirometry.

We wish to thank Mr J F Bannister from the department of medical physics, for his invaluable help in writing the computer program used to calculate the oximetry data and the nursing staff on the Regional Cystic Fibrosis Unit.

1 Moss AJ. The cardiovascular system in cystic fibrosis. Pediatrics 1982; 70: 728-41.

2 Goldring RM, Fishman AP, Turino GM, Cohen HI, Denning CR, Anderson DH. Pulmonary hypertension and cor pulmonale in cystic fibrosis of the pancreas. f Pediatr 1964; 65: 501-24.

3 Fletcher EC, Luckett RA, Miller T, Costarangos C, Kutka $\mathrm{N}$, Fletcher JG. Pulmonary vascular haemodynamics in chronic lung disease patients with and without oxyhaemochronic lung disease patients with and without oxyhaemo-
globin desaturation during sleep. Chest 1989; 95: 157-66.

4 Muller NL, Francis PW, Gurwitz D, Levison H, Bryan AC. Mechanism of hemoglobin desaturation during rapid eye movement sleep in normal subjects and in patients with cystic fibrosis. Am Rev Respir Dis 1980; 121: 463-9.

5 Francis PW, Muller NL, Gurwitz D, Milligan DWA Levinson H, Bryan AC. Hemoglobin desaturation - its occurrence during sleep in patients with cystic fibrosis. Am ₹ Dis Child 1980; 134: 734-40.

6 Stokes DC, McBride JT, Wall MA, Erba G, Streider DJ. Sleep hypoxemia in young adults with cystic fibrosis. $A m$ f Dis Child 1980; 134: 741-3.

7 Spier S, Rivlin J, Hughes D, Levison $H$. The effect of oxygen on sleep, blood gases and ventilation in cystic fibrosis. Am Rev Respir Dis 1984; 129: 712-8.

8 Tepper RS, Skatrud JB, Dempsey JA. Ventilation and oxygenation changes during sleep in cystic fibrosis. Ches 1983; 84: 388-93.

9 Versteegh FGA, Neijens HJ, Bogaard JM, Stam H, Robijn RJ, Kerrebijn KF. Relationship between pulmonary function, $\mathrm{O}_{2}$ saturation during sleep and exercise, and exercise responses in children with cystic fibrosis. $A d v$ Cardiol 1986; 35: 151-5.

10 Versteegh FGA, Bogaard J, Neijens HJ, Kerrebijn KF. Relationship between airway obstruction, desaturation Relationship between airway obstruction, desaturation
during exercise and nocturnal hypoxaemia in cystic during exercise and nocturnal hypoxaemia

11 Montgomery M, Wiebicke W, Bibi H, Pagtakhan RD, Pasterkamp $\mathrm{H}$. Home measurement of oxygen saturation during sleep in patients with cystic fibrosis. Pediat Pulmonol 1989; 7: 29-34.

12 Coffey MJ, FitzGerald MX, McNicholas WT. Comparison of oxygen desaturation during sleep and exercise in patients with cystic fibrosis. Chest 1991; 100: 659-62.

13 Shwachman H, Kulczycki LL. Long term study of one hundred five patients with cystic fibrosis. Am $\mathcal{F}$ Dis Child 1958; 96: 6-15.

14 Chrispin AR, Norman AP. The systematic evaluation of the chest radiograph in cystic fibrosis. Pediatr Radiol 1974; 2 101-5. 
15 Conway SP, Miller MG, Ramsden C, Littlewood JM. Intensive treatment of pseudomonas chest infections in cystic fibrosis: a comparison of tobramycin and ticarcillin and netilmicin and ticarcillin. Acta Paediatr Scand 1985; 74: 107-13.

16 Beaudry PH, Marks MI, McDougall D, Desmond $\mathrm{K}$ Raugel RJ. Is anti-pseudomonas therapy warranted in acute respiratory exacerbations in children with cystic fibrosis? F Pediatr 1980; 97: 144-7.

17 Warley ARH, Mitchell JH, Stradling JR. Evaluation of the Ohmeda 3700 pulse oximeter. Thorax 1987; 42: 892-6.

18 Schoni MH, Hurliman $\mathrm{R}$, Bruderer $\mathrm{K}$. Pulse oximetry, digital clubbing and cystic fibrosis. Lancet 1987; i: 1317.

19 Prowse K, Allen MB. Problems in practice: sleep apnoea. British fournal of Diseases of the Chest 1988; 82: 329-40.
20 Birchfield RI, Seiker HO, Heyman A. Alterations in respiratory function during natural sleep. $f \mathrm{Lab}$ Clin $\mathrm{Med}$ 1959; 54: 216-22.

21 Block AJ, Boysen PG, Wynne JW, Hunt LA. Sleep apnoea, hypopnoea and oxygen desaturation in normal subjects. NEngl $\Im$ Med 1979; 300: 513-7.

22 Stradling JR, Thomas G, Warley ARH, Williams $P$, Freeland A. Effect of adenotonsillectomy on nocturnal hypoxaemia, sleep disturbance and symptoms in snoring children. Lancet 1990; 335: 249-53.

23 Agnew HW, Webb WB, Williams RL. The first night effect: an EEG study of sleep. Psychopharmacology 1966; 2: 263-6. 24 Levi-Valensi P, Weitzenblum E, Pendinielli JL, Racineux JL Duwoos $\mathrm{H}$. Three month follow up of arterial blood gas determinations in candidates for long term oxygen therapy. Am Rev Respir Dis 1986; 133: 547-51. 